\title{
Water-based exercise in COPD with physical comorbidities: a randomised controlled trial
}

\author{
Renae J. McNamara*,\#, , Zoe J. McKeough*, David K. McKenzie ${ }^{\#}$ and \\ Jennifer A. Alison*,+
}

ABSTRACT: Land-based exercise is often difficult for people with chronic obstructive pulmonary disease (COPD) who have coexisting obesity or musculoskeletal or neurological conditions. This randomised controlled trial aimed to determine the effectiveness of water-based exercise training in improving exercise capacity and quality of life compared to land-based exercise training and control (no exercise) in people with COPD and physical comorbidities.

Participants referred to pulmonary rehabilitation were randomly allocated to a water-based exercise, land-based exercise or the control group. The two exercise groups trained for 8 weeks, completing three sessions per week.

45 out of 53 participants (mean \pm SD age $72 \pm 9$ years; forced expiratory volume in 1 s $59 \pm 15 \%$ predicted) completed the study. Compared to controls, water-based exercise training significantly increased 6-min walking distance, incremental and endurance shuttle walk distances, and improved Chronic Respiratory Disease Questionnaire (CRDQ) dyspnoea and fatigue. Compared to land-based exercise training, water-based exercise training significantly increased incremental shuttle walk distance (mean difference $39 \mathrm{~m}, 95 \% \mathrm{Cl}$ 5-72 $\mathrm{m}$ ), endurance shuttle walk distance (mean difference $228 \mathrm{~m}, 95 \% \mathrm{Cl}$ 19-438 $\mathrm{m}$ ) and improved CRDQ fatigue.

Water-based exercise training was significantly more effective than land-based exercise training and control in increasing peak and endurance exercise capacity and improving aspects of quality of life in people with COPD and physical comorbidities.

KEYWORDS: Chronic obstructive pulmonary disease, comorbidity, exercise, walking, water

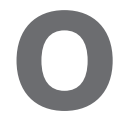
ne of the most effective strategies for the management of chronic obstructive pulmonary disease (COPD) is land-based exercise training as part of integrated pulmonary rehabilitation. Land-based exercise training improves exercise capacity and quality of life [1]. However, the majority of people with COPD referred to pulmonary rehabilitation are in the older age category [2], and a high proportion have physical comorbidities, such as skeletal muscle abnormalities, cerebrovascular disease, arthroses and obesity [3-5], which may limit their ability to participate in land-based exercise training. High drop-out rates from land-based exercise training ranging from 14 to $66 \%$ have been reported, as completion of land-based exercise training is often difficult or impossible for patients with comorbid conditions $[2,6]$. The limitations such physical comorbidities place on a person's ability to perform land-based exercise training may considerably reduce the effectiveness of this form of training. People with COPD and physical comorbidities are usually excluded from research studies of the effects of exercise training and therefore the findings of such studies cannot be extrapolated to them. Waterbased exercise training is effective in the management of people with a primary diagnosis of musculoskeletal conditions, including rheumatoid and osteo-arthritis and chronic low back pain, as well as some neurological conditions, peripheral vascular disease and obesity [7-9]. For people with COPD and a secondary diagnosis of these conditions, water-based exercise training may provide an alternative mode of training.

Water-based exercise training had previously been thought to be unsafe for people with COPD due to the increased chest wall pressure and increased cardiac output that result from immersion in water [10]. Despite lung function restriction in water, no adverse events have been reported from a single exercise session in water and oxygen saturation was maintained, even in
AFFILIATIONS

${ }^{*}$ Clinical and Rehabilitation Sciences, University of Sydney, Lidcombe,

\#Dept of Respiratory and Sleep Medicine, Prince of Wales Hospital, Randwick,

"Dept of Physiotherapy, Prince of Wales Hospital, Randwick, and +Dept of Physiotherapy, Royal Prince Alfred Hospital, Camperdown, Australia.

CORRESPONDENCE

R.J. McNamara

Dept of Physiotherapy

Prince of Wales Hospital

Barker Street

Randwick

NSW 2031

Australia

E-mail: renae.mcnamara@

sesiahs.health.nsw.gov.au

Received:

Feb 262012

Accepted after revision:

Sept 072012

First published online:

Sept 202012

European Respiratory Journal

Print ISSN 0903-1936

Online ISSN 1399-3003 
those with severe COPD [11]. Only one prospective study has examined the effect of water-based exercise training in COPD [12]. In a semi-randomised trial (randomisation by geographical distance from training venue), WADELL et al. [12] reported significantly greater improvements in endurance walking capacity and self-reported quality of life following 12 weeks of water-based exercise training compared to land-based exercise training. Importantly, people with any physical comorbidity that could impact on exercise performance were excluded from participating in this study. In contrast, we postulated that people with COPD and a physical comorbidity would benefit more from water-based exercise training as these people were less likely to be able to complete or derive benefit from land-based exercise training.

The purpose of this study was to determine the effects of water-based exercise training primarily on exercise capacity and secondarily on health-related quality of life and respiratory muscle strength, compared with land-based exercise training or no exercise training in people with COPD and physical comorbidities. We hypothesised that water-based exercise training in people with COPD and a physical comorbidity would be more effective in improving exercise capacity, quality of life and respiratory muscle strength than land-based exercise training or no exercise training.

\section{METHODS}

\section{Participants}

Patients who were referred to outpatient pulmonary rehabilitation at an Australian tertiary public hospital were included in the study if they had a confirmed diagnosis of COPD according to Global Inititative for Obstructive Lung Disease (GOLD) criteria (forced expiratory volume in $1 \mathrm{~s}$ (FEV1)/ forced vital capacity (FVC) $<70 \%$ ) that was in a stable phase and the presence of one or more physical comorbidities (including musculoskeletal conditions affecting lumbar spine or lower limbs, one or more lower limb joint replacement restricting mobility and/or range of motion, or peripheral vascular disease, neurological conditions such as a stroke or obesity with body mass index $(\mathrm{BMI}) \geqslant 32 \mathrm{~kg} \cdot \mathrm{m}^{-2}$ ). Diagnosis of the physical comorbidity was based on medical referral, patient history and physical examination. Subjects were excluded if they had unstable cardiac disease, contraindications to water-based therapy [13], such as uncontrollable incontinence or open wounds, had completed pulmonary rehabilitation in the past 12 months or were currently attending an exercise programme, had cognitive decline or were unable to understand oral and written English. Subjects using supplemental oxygen were included.

\section{Study design and randomisation}

This study was a prospective randomised controlled trial with assessor blinding. Participants satisfying the eligibility criteria were randomised by an investigator external to the study using a web-based computer-generated sequence (www.randomization.com). Concealed allocation was achieved using opaque envelopes. Participants were randomised to one of three groups: water-based exercise training, land-based exercise training or control (no exercise). Randomisation was stratified according to the limiting factor in the 6-min walk test (6MWT) (that is, breathlessness or physical comorbidity) and BMI $\left(\geqslant 32 \mathrm{~kg} \cdot \mathrm{m}^{-2}\right)$. Due to the nature of the exercise interventions, it was not possible to blind the therapist or participants to their allocation. Ethics approval was obtained by the South Eastern Sydney Area Health Service Northern Network Human Research Ethics Committee and written informed consent was obtained. The trial was registered with the Australian New Zealand Clinical Trials Registry (ACTR number: ACTRN012606000408583).

\section{Measurements}

The primary outcome measure was endurance exercise capacity measured by the endurance shuttle walk test (ESWT).

Participants attended two visits within a 7-day period both at baseline and immediately post-intervention to undergo the following measurements.

\section{Pulmonary function tests}

Spirometry (FEV1 and FVC), diffusing capacity of the lung for carbon monoxide, static lung volumes by body plethysmography, and maximum inspiratory and expiratory mouth pressure (MIP and MEP, respectively) were performed according to recommended guidelines [14-17] following the administration of inhaled salbutamol via a spacer device.

\section{Exercise tests}

Exercise capacity was assessed by the self-paced 6MWT, and the externally paced incremental shuttle walk test (ISWT) and ESWT, according to standardised guidelines [18-20]. Two tests were performed for each walk test, separated by a period of $\geqslant 30 \mathrm{~min}$, over two visits within 7 days of each other. The order of the three tests at each visit was 6MWT then ISWT followed by ESWT. The better result for each test was used in the analysis. Self-reported dyspnoea and exertion were measured on the modified Borg 0-10-category ratio scale for dyspnoea and perceived exertion [21].

\section{Health-related quality of life and anxiety and depression}

Participants completed the self-reported Chronic Respiratory Disease Questionnaire (CRDQ) [22] and the Hospital Anxiety and Depression questionnaire [23].

\section{Intervention}

The water-based and land-based exercise training groups attended three 60 -min sessions a week of supervised exercise led by the same experienced physiotherapist for 8 weeks, with a maximum of 12 participants per session. Exercise in water and on land was matched as closely as possible for intensity, duration and muscle groups trained, considering the different exercise media (notably the increased resistance experienced during movement in water) (table 1). Participants were encouraged to exercise at an intensity rating of three to five on the modified Borg scale for dyspnoea and perceived exertion. Training intensity was measured three times during each exercise session and the mean value recorded. If the intensity reported was below three, participants were encouraged to increase their intensity as detailed in table 1 . The land-based exercise training group exercised in a temperaturecontrolled hospital gymnasium and participants walked at an intensity of $80 \%$ of the average $6 \mathrm{MWT}$ speed either over ground or on a treadmill. The water-based exercise training group exercised in a hospital hydrotherapy pool (depth 
TABLE 1 Land-based and water-based exercise training programmes

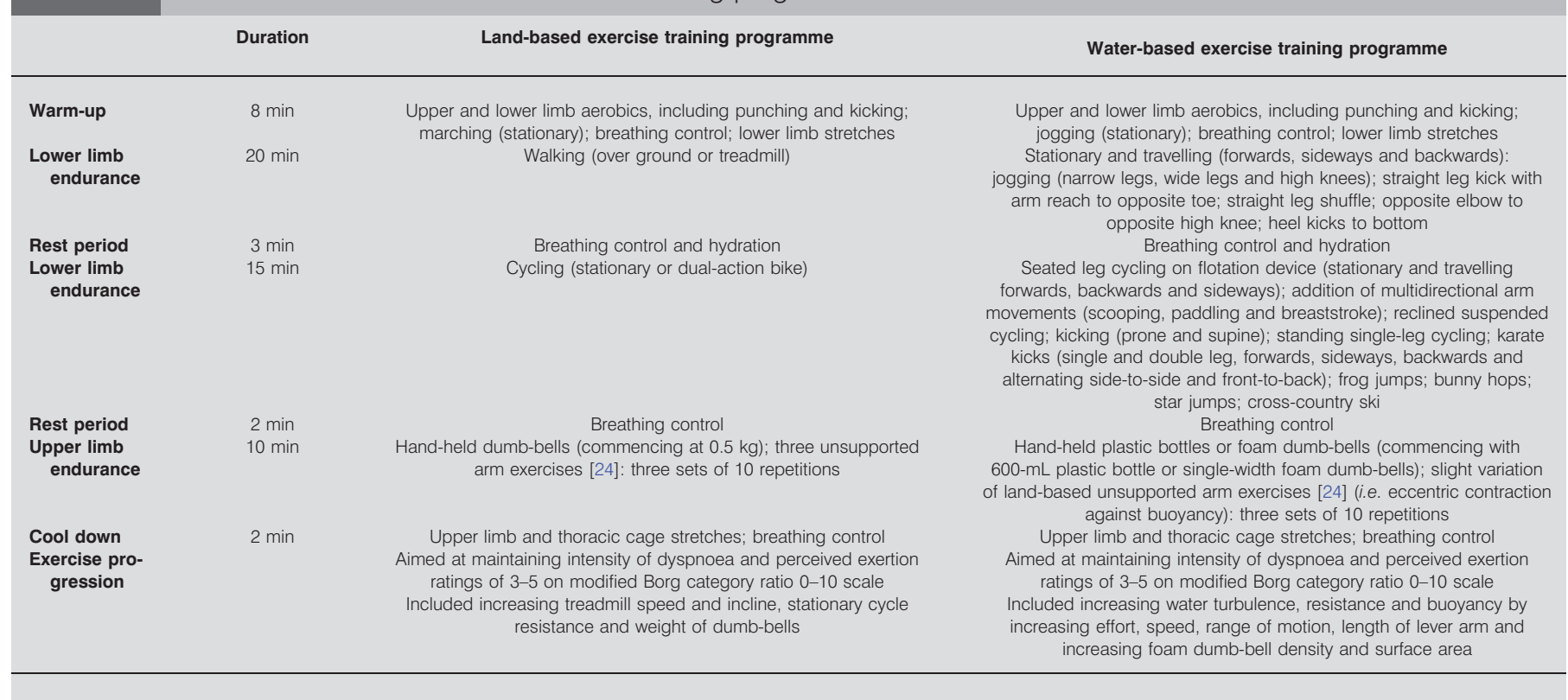

graduating from $1.1 \mathrm{~m}$ to $1.6 \mathrm{~m}$; length $18 \mathrm{~m}$; width $6 \mathrm{~m}$ ) with water temperature of $34^{\circ} \mathrm{C}$, air temperature of $30^{\circ} \mathrm{C}$ and relative air humidity of $30 \%$. Water-based exercise training participants were able to choose the most comfortable level of water immersion in the standing position to perform the majority of exercises, which was always between the xiphisternum and the clavicles for each participant. Exercises in water and on land were performed as detailed in table 1. Control group participants received usual medical care and no exercise training. They were asked not to alter their exercise level over the study period.

\section{Sample size}

A total of 54 participants (18 per group) were required to ensure that 48 participants (16 per group) completed the study, allowing for $10 \%$ loss to follow-up. 48 participants were sufficient to detect as significant, at the 5\% level (two-sided), a 203-m difference in the mean ESWT distance between the water-based and land-based exercise training groups. This assumed a standard deviation of $200 \mathrm{~m}$ as previously reported [25]. A 203-m difference in ESWT distance is considered the minimum important difference [25]. This sample size also provided sufficient power for the comparison of the waterbased exercise training group to the control group and the land-based exercise training group to the control group.

\section{Statistical analysis}

Statistical analysis was performed using SPSS Statistics 17.0 (SPSS Inc., Chicago, IL, USA). Within-group comparisons were examined using a paired t-test. Statistical significance was determined from post hoc comparisons using least significant differences. ANCOVA was used to conduct between-group comparisons of outcomes after adjusting for pre-intervention values [26]; however, there was no adjustment of p-values for multiple comparisons to avoid Type II errors. The level of statistical significance was set at $p<0.05$ for all analyses.
Uncertainty about the size of the mean differences between groups was quantified with $95 \%$ confidence intervals.

\section{RESULTS}

Out of 60 consecutive patients referred to pulmonary rehabilitation with COPD and physical comorbidities, 53 were enrolled in the trial. 45 participants completed the study and were included in the analyses (fig. 1). Baseline characteristics were similar between the three groups (table 2).

Compliance with exercise group attendance was high, with participants allocated to the water-based exercise training group attending a mean \pm SD of $21 \pm 2$ sessions out of a total of 24 sessions and participants in the land-based exercise training group attending $19 \pm 4$ out of 24 sessions, with no statistical difference in attendance between groups $(p=0.11)$. Five participants randomised to the land-based exercise training group failed to complete the study. Four of these participants experienced an exacerbation of their primary comorbid condition, which caused them to drop out of the study, and one participant suffered acute-on-chronic knee pain during treadmill walking training and chose to drop out of the study. Three participants randomised to the water-based exercise training group failed to complete the study; however, none of these withdrawals were related to exacerbations of their comorbid conditions. One participant suffered an accidental skin tear to the lower leg from another participant and chose to drop out of the study and the remaining two participants discontinued attending due to general fatigue. There were no statistically significant differences in baseline characteristics between participants completing the study and participants who dropped out of the study.

On the modified Borg 0-10-category ratio scale for dyspnoea, participants in the water-based exercise training group trained at an average self-reported intensity of $4.5 \pm 0.5$ over the 8week training period compared to $3.0 \pm 0.5$ in the land-based exercise training group $(\mathrm{p}<0.001)$. On the modified Borg 


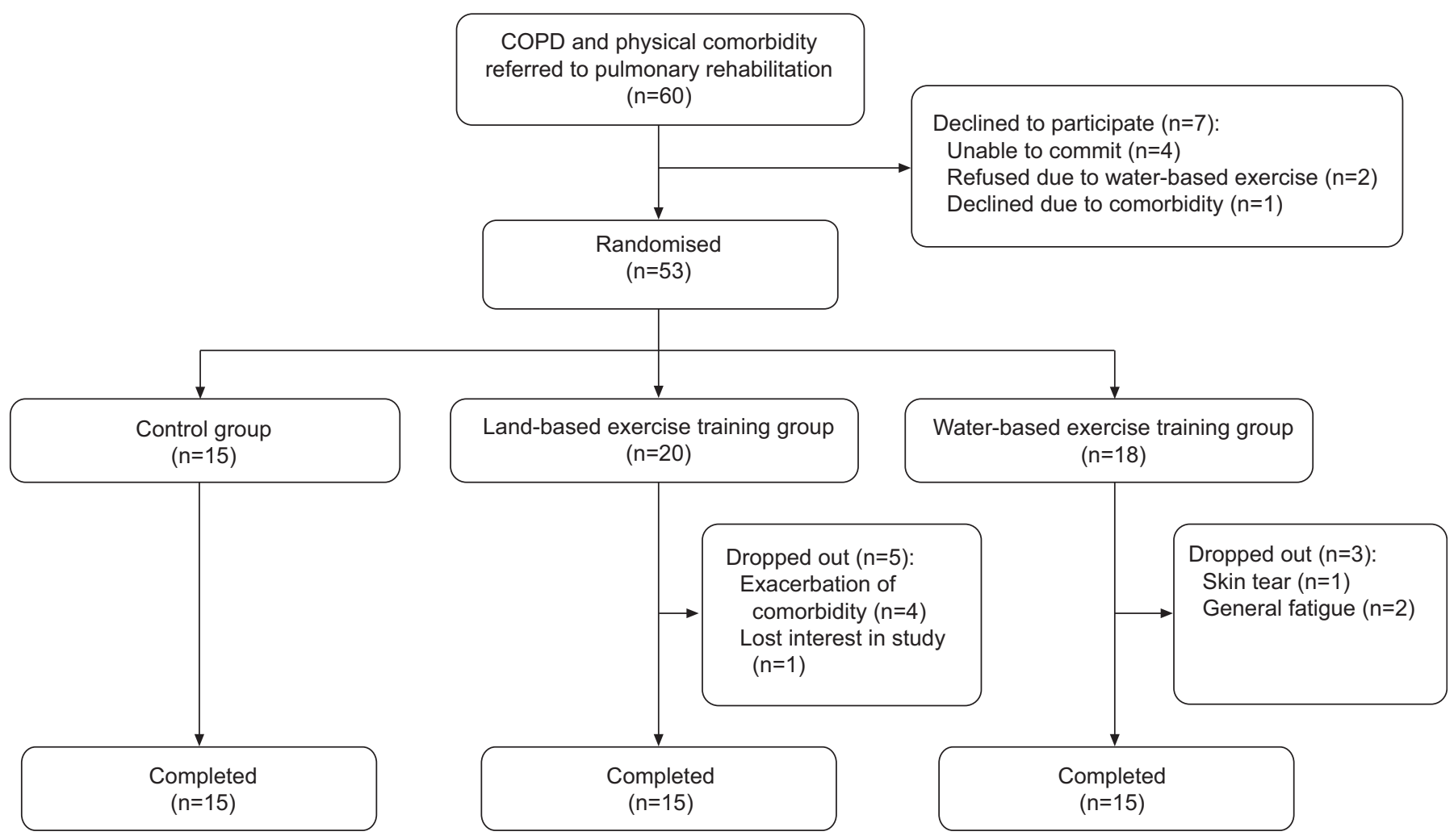

FIGURE 1. Participant flow. COPD: chronic obstructive pulmonary disease.

$0-10$-category ratio scale for perceived exertion, participants in the water-based exercise training group trained at an average self-reported intensity of $4.5 \pm 0.5$ over the 8 -week training period compared to $3.5 \pm 0.5$ in the land-based exercise training group $(p<0.001)$. The mean \pm SD training intensities achieved each week over the 8-week training period in the two exercise training groups are illustrated in figure 2. Aside from the dyspnoea ratings in the first 2 weeks of training in the landbased exercise training group, both training groups trained at the desired intensity for dyspnoea and perceived exertion.

\section{Exercise capacity}

The mean changes in exercise test outcomes from baseline to immediately post-intervention are reported in table 3. Significant within-group improvements in 6MWT distance occurred following training in the water-based and land-based exercise training groups (table 3), and a significant betweengroup difference was found when each of the exercise groups were compared with the control group (table 4). The waterbased exercise training group was the only group to achieve a significant within-group change in the ISWT and ESWT following training (table 3). Compared to land-based exercise training and the control group, water-based exercise training significantly increased incremental shuttle walk distance and endurance shuttle walk distance (table 4). The predominant limiting factors in walking test performance for all participants were dyspnoea and musculoskeletal complaints. In the waterbased exercise training group at baseline assessment, musculoskeletal pain was the limiting factor in $53 \%$ and $60 \%$ of participants in the ISWT and ESWT, respectively, while dyspnoea was the limiting factor in $40 \%$ and $33 \%$ of participants in the ISWT and ESWT, respectively. At final assessment, musculoskeletal pain was the limiting factor in $60 \%$ and $53 \%$ of participants in the ISWT and ESWT, respectively, while dyspnoea was the limiting factor in $27 \%$ of participants in both the ISWT and ESWT. Following waterbased exercise training, there was no significant change to the ISWT end-test dyspnoea score (both baseline mean \pm SD and follow-up mean \pm SD $4 \pm 1$ ) or the ESWT end-test dyspnoea score (baseline mean \pm SD $4.5 \pm 2$ and follow-up mean \pm SD $4 \pm 2$ ).

\section{Health-related quality of life and anxiety and depression}

A significant between-group difference was found between the water-based and land-based exercise training groups in the CRDQ fatigue domain and between the water-based exercise training and control group in dyspnoea and fatigue domains (table 4). No between-group differences were found in anxiety or depression scores following exercise training.

\section{Respiratory muscle tests}

Compared to control, water-based exercise training significantly increased MIP (table 4). There were no significant between-group differences in MEP.

\section{DISCUSSION}

This is the first prospective randomised controlled study of the effects of water-based exercise training in people with a primary diagnosis of COPD and secondary physical comorbidities. This study showed that water-based exercise training in people with COPD and physical comorbidities improved peak and endurance exercise capacity and the fatigue domain of the CRDQ significantly more than an equivalent land-based 
TABLE 2 Baseline characteristics of study participants

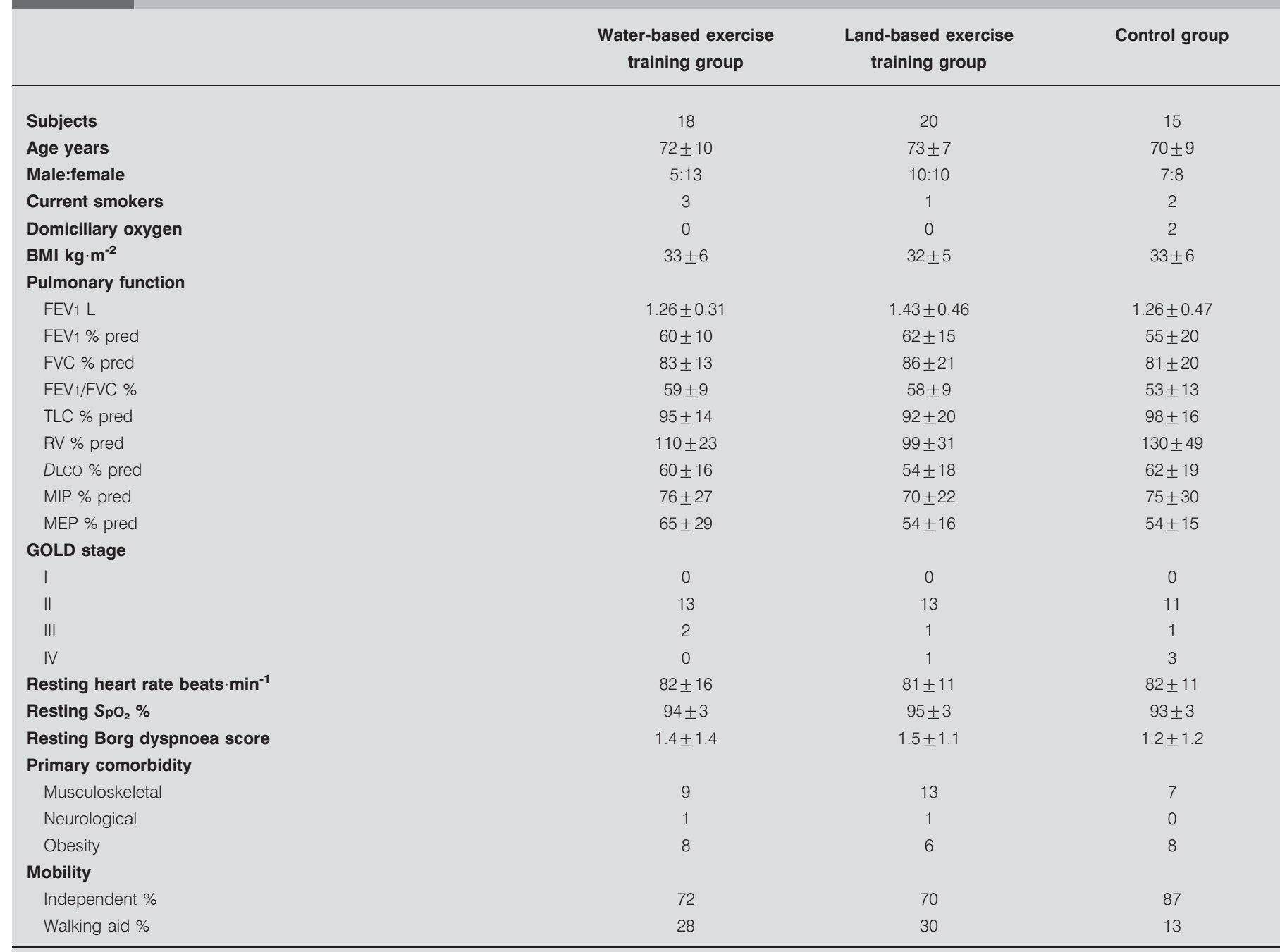

Data are presented as $\mathrm{n}$ or mean $\pm \mathrm{SD}$, unless otherwise stated. All values are post-bronchodilator. BMI: body mass index; FEV1: forced expiratory volume in $1 \mathrm{~s}$; \% pred.: \% predicted; FVC: forced vital capacity; TLC: total lung capacity; RV: residual volume; DLCO: diffusing capacity of the lung for carbon monoxide; MIP: maximal inspiratory mouth pressure; MEP: maximal expiratory mouth pressure; GOLD: Global Initiative for Chronic Obstructive Lung Disease; $\mathrm{SpO}_{2}$ : arterial oxygen saturation measured by pulse oximetry.

exercise training programme or a control group of no exercise training. Furthermore, water-based exercise training resulted in significant improvements in inspiratory muscle strength and the dyspnoea domain of the CRDQ compared to no exercise training.

Water-based exercise training was shown to be more effective in improving exercise capacity than land-based exercise training and no exercise training. The between-group differences for the ISWT and ESWT surpassed the minimum clinically important differences for both these tests in all group comparisons [25, 27]. However, there was no significant difference in 6MWT distance between the water-based and land-based exercise training groups. Only one previous prospective study has compared water-based exercise training with land-based exercise training in people with COPD, showing a between-group difference of $20 \mathrm{~m}$ in ISWT distance and $164 \mathrm{~m}$ in ESWT distance in favour of water-based exercise training [12]. However, in this study subjects with physical comorbidities were excluded and the study was only semirandomised, with groups determined by geographical distance from training venue rather than by true randomisation [12]. A key difference is that our study examined people with physical comorbidities and had stronger methodological features of randomisation and assessor blinding. Interestingly, our results demonstrated a greater between-group difference in exercise capacity of $39 \mathrm{~m}$ in ISWT distance and $228 \mathrm{~m}$ in ESWT distance in favour of water-based exercise training, suggesting that people with COPD and physical comorbidities may be better responders to water-based exercise training than those with COPD alone. An interesting finding was that although the water- and land-based groups increased 6MWT distance, there was no significant difference between the groups, despite a significant between-group difference in ESWT in favour of the 

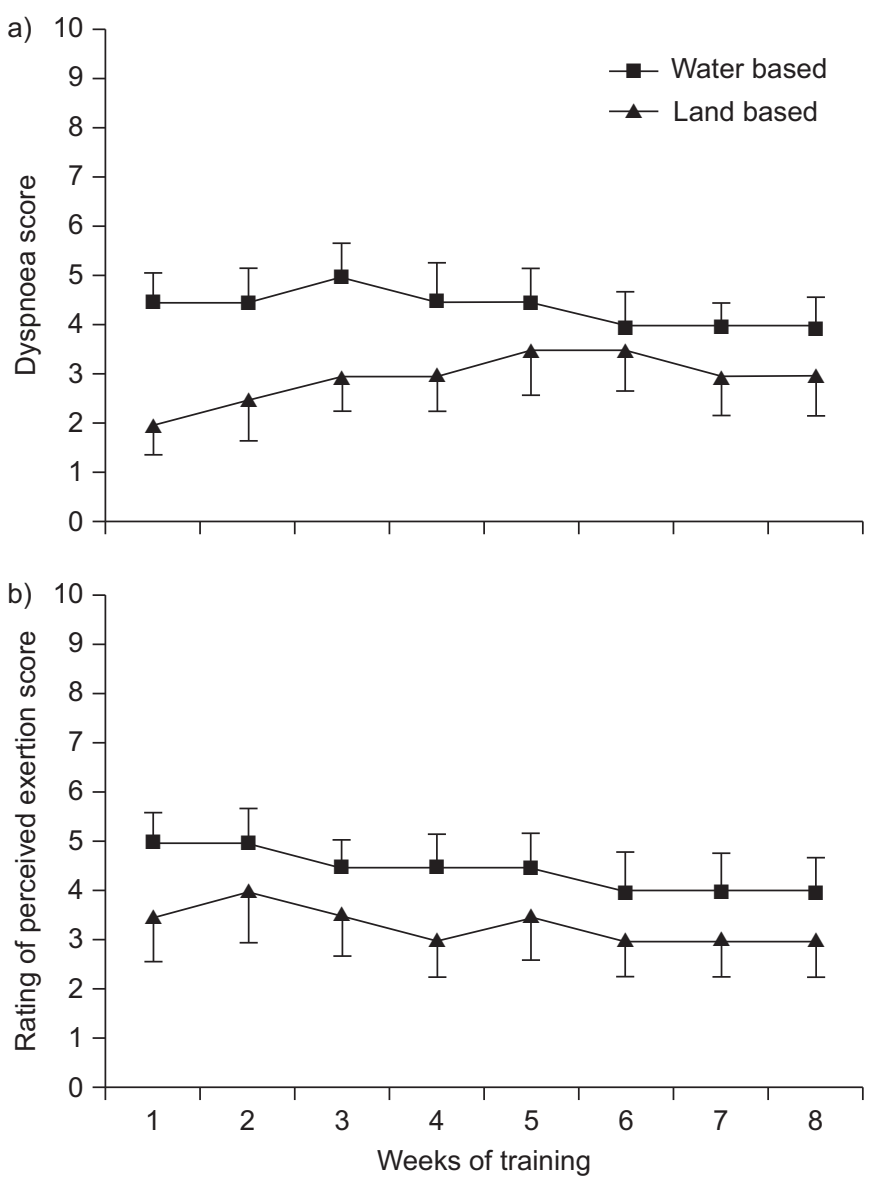

FIGURE 2. Training intensities over the 8-week training period for water-based and land-based training groups according to a) dyspnoea score and b) perceived exertion score

water-based training. This finding is most probably related to the constructs of the tests. For an improvement in 6MWT the participant needs to walk faster, whereas for improvement in the ESWT the participant needs to walk at the same constant speed for longer. In people with physical comorbid conditions, training in water may not improve walking speed as much as endurance walking capacity, thus the ESWT may be more sensitive to change than the $6 \mathrm{MWT}$ following water-based exercise training in people with physical comorbidities.
While the water-based exercise training was shown to be very effective compared to land-based exercise training, it is interesting to note that in this cohort of people with COPD and physical comorbidities, land-based exercise training was still effective compared to the control of no exercise. Although there is high-level evidence from randomised controlled trials that there are benefits of land-based exercise training in improving exercise capacity in people with COPD, no previous studies have specifically examined people with COPD and physical comorbidities. This work extends the previous findings and demonstrates that land-based exercise training can be effective for those with COPD and physical comorbidities. However, our findings of the superiority of water-based exercise training compared to land-based exercise training suggest that the water medium has added benefits. The unique water properties of buoyancy to support body weight [28], combined with resistance and turbulence to increase exercise intensity [28], as well as the proposed effects of warm water on blood flow to muscle [28], may have enabled our population of patients with COPD and physical comorbidities to exercise at a higher intensity by reducing the impact of their physical comorbidity on exercise. Some indirect support for higher exercise intensity in water compared to land was provided by the subjective symptom responses to training shown in figure 2.

Our data shows that water-based exercise training achieved important outcomes for daily life in patients with COPD, with a reduction in CRDQ dyspnoea and fatigue domain scores compared to no training, as well as a significant reduction in CRDQ fatigue domain score compared to land-based exercise training. As the CRDQ is a disease-specific health-related quality of life questionnaire, these improvements demonstrate that water-based exercise training in people with COPD and physical comorbidities has a greater effect than just improving the physical comorbid condition, and actually improves health-related quality of life and management of COPD.

It has been proposed previously that immersion in water may not be tolerated by people with COPD as the hydrostatic pressure placed on the chest wall may increase the work of breathing [11]. However, people with COPD in our study tolerated the water environment well with a high attendance rate, fewer drop-outs than the land-based exercise training group and the ability to train at the desired intensity. It is likely that the hydrostatic pressure placed on the chest wall provided an added load to the

TABLE 3 Within-group changes in exercise capacity outcomes

\begin{tabular}{|c|c|c|c|c|c|c|c|c|c|c|c|}
\hline \multicolumn{4}{|c|}{ Water-based exercise training group ${ }^{\#}$} & \multicolumn{4}{|c|}{ Land-based exercise training group ${ }^{\#}$} & \multicolumn{4}{|c|}{ Control group $\#$} \\
\hline Baseline & $\begin{array}{c}\text { Follow- } \\
\text { up }\end{array}$ & Change & p-value & Baseline & Follow-up & Change & p-value & Baseline & $\begin{array}{c}\text { Follow- } \\
\text { up }\end{array}$ & Change & p-value \\
\hline $271 \pm 153$ & $591 \pm 367$ & $321(123-518)$ & 0.004 & $222 \pm 149$ & $339 \pm 299$ & $117(-3-236)$ & 0.055 & $396 \pm 423$ & $345 \pm 261$ & $-50(-240-140)$ & 0.580 \\
\hline $349 \pm 91$ & $397 \pm 68$ & $48(27-70)$ & $<0.001$ & $300 \pm 142$ & $343 \pm 131$ & $43(22-63)$ & 0.001 & $364 \pm 88$ & $347 \pm 94$ & $-16(-34-1)$ & 0.060 \\
\hline $186 \pm 93$ & $235 \pm 96$ & $49(26-73)$ & 0.001 & $165 \pm 119$ & $178 \pm 102$ & $13(-16-43)$ & 0.350 & $195 \pm 96$ & $195 \pm 104$ & $-1(-24-22)$ & 0.951 \\
\hline
\end{tabular}

Data are presented as mean \pm SD or mean $(95 \% \mathrm{CI})$, unless otherwise stated. ESWT: endurance shuttle walk test; $6 \mathrm{MWT}$ : 6-min walk test; ISWT: incremental shuttle walk test. ${ }^{\#}: \mathrm{n}=15$; ${ }^{\bullet}$ : from paired t-test. 


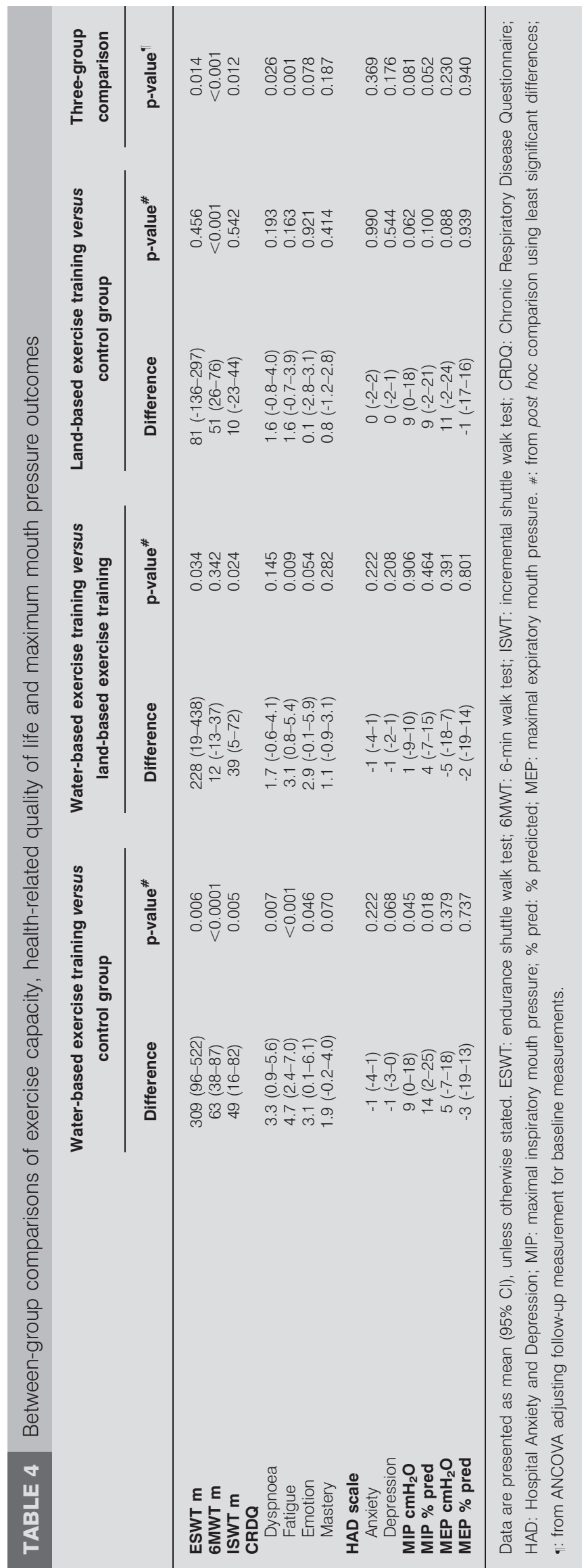

inspiratory muscles of respiration, which resulted in a training stimulus as indicated by an increase in MIP compared to control. This is the first study to show a positive effect of water-based exercise training on inspiratory muscle strength.

A limitation of this study was that no physiological data were available to verify that the improvements in exercise capacity were specifically related to adaptation of peripheral muscles. However, dyspnoea scores were similar to pre-training values at the end of the ISWT and ESWT after water-based exercise training, in spite of greater work capacity being achieved. This result provides some evidence to suggest that changes in peripheral muscle may have occurred, such as less lactate production for the same work providing less stimulus to ventilation and hence less dyspnoea. The fact that exercise was terminated at the same level of dyspnoea in the two walk tests is consistent with a large body of evidence concerning physiological limits to exercise [29]. Further studies should be conducted to confirm that these changes in exercise capacity following water-based exercise training are the result of physiological training effects. Furthermore, as the majority of participants in this study were classified as GOLD stage II, these results may not apply to people with severe COPD and thus further investigation is required.

\section{Conclusion}

This randomised controlled trial found that water-based exercise training was effective in improving exercise capacity and some aspects of health-related quality of life in people with COPD and physical comorbidities compared to land-based exercise training and no exercise training. This study provides compelling evidence for water-based exercise training as an alternative to land-based exercise training to improve function on land in people with COPD and physical comorbidities.

\section{SUPPORT STATEMENT}

This study received funding from the Physiotherapy Research Foundation (grant number S07-011).

\section{CLINICAL TRIAL}

This study is registered at Australian New Zealand Clinical Trials registry (www.anzctr.org.au) with identifier number ACTRN012606000408583.

\section{STATEMENT OF INTEREST}

None declared.

\section{ACKNOWLEDGEMENTS}

The authors acknowledge and thank: Mary Santos (Dept of Physiotherapy, Prince of Wales Hospital, Randwick, Australia) for her assistance with data collection; Amir Ghanbari, Sandra Jeffery and Garry McClenaghan (Dept of Respiratory Medicine, Royal Prince Alfred Hospital, Camperdown, Australia) for their assistance with respiratory function testing; Mark Elkins (Dept of Respiratory Medicine, Royal Prince Alfred Hospital) and Jennifer Peat (Australian Catholic University, North Sydney, Australia) for review and advice on statistical analysis; the staff of the Respiratory and Sleep Medicine and Physiotherapy Departments, Prince of Wales Hospital, Randwick, Australia for support; and the patients who participated in the study.

\section{REFERENCES}

1 Lacasse Y, Goldstein R, Lasserson TJ, et al. Pulmonary rehabilitation for chronic obstructive pulmonary disease. Cochrane Database Syst Rev 2006; 4: CD003793. 
2 Keating A, Lee A, Holland AE. What prevents people with chronic obstructive pulmonary disease from attending pulmonary rehabilitation? A systematic review. Chron Respir Dis 2011; 8: 89-99.

3 Fabbri LM, Luppi F, Beghe B, et al. Complex chronic comorbidities of COPD. Eur Respir J 2008; 31: 204-212.

4 Crisafulli E, Costi S, Luppi F, et al. Role of comorbidities in a cohort of patients with COPD undergoing pulmonary rehabilitation. Thorax 2008; 63: 487-492.

5 Franssen FME, O'Donnell DE, Goossens GH, et al. Obesity and the lung: 5. Obesity and COPD. Thorax 2008; 63: 1110-1117.

6 Garrod R, Marshall J, Barley E, et al. Predictors of success and failure in pulmonary rehabilitation. Eur Respir J 2006; 27: 788-794.

7 Geytenbeek J. Evidence of effective hydrotherapy. Physiotherapy 2002; 88: 514-529.

8 Carpentier PH, Satger B. Randomised trial of balneotherapy associated with patient education in patients with advanced chronic venous insufficiency. J Vasc Surg 2009; 49: 163-170.

9 Gappmaier E, Lake W, Nelson AG, et al. Aerobic exercise in water versus walking on land: effect on indices of fat reduction and weight loss of obese women. J Sports Med Phys Fitness 2006; 46: 564-569.

10 Arborelius M, Balldin UI, Lilja B, et al. Hemodynamic changes in man during immersion with head above water. Aerosp Med 1972. 43: 592-598.

11 Perk J, Perk L, Boden C. Cardiorespiratory adaptation of COPD patients to physical training on land and in water. Eur Respir J 1996; 9: 248-252.

12 Wadell K, Sundelin G, Henriksson-Larsen K, et al. High intensity physical group training in water - an effective training modality for patients with COPD. Respir Med 2004; 98: 428-438.

13 Larsen J, Pryce M, Harrison J, et al. Guidelines for Physiotherapists Working in and/or Managing Hydrotherapy Pools. Melbourne, Australian Physiotherapy Association, 2002.

14 Standardization of spirometry, 1994 update. American Thoracic Society. Am J Respir Crit Care Med 1995; 152: 1107-1136.

15 American Thoracic Society. Single-breath carbon-monoxide diffusing capacity (transfer factor). Recommendations for a standard technique - 1995 update. Am J Respir Crit Care Med 1995; 152: 2185-2198.
16 Blonshine S, Foss C, Ruppel G, et al. American Association for Respiratory Care. AARC clinical practice guidelines: body plethysmography - 2001 revision and update. Respir Care 2001; 46: 506-513.

17 American Thoracic Society, European Respiratory Society. ATS/ ERS statement on respiratory muscle testing. Am J Respir Crit Care Med 2002; 166: 518-624.

18 ATS Committee on Proficiency Standards for Clinical Pulmonary Function Laboratories. ATS statement: guidelines for the sixminute walk test. Am J Repir Crit Care Med 2002; 166: 111-117.

19 Singh SJ, Morgan MD, Scott S, et al. Development of a shuttle walking test of disability in patients with chronic airways obstruction. Thorax 1992; 47: 1019-1024.

20 Revill SM, Morgan MD, Singh SJ, et al. The endurance shuttle walk: a new field test for the assessment of endurance capacity in chronic obstructive pulmonary disease. Thorax 1999; 54: 213-222.

21 Borg GAV. Psychophysical bases of perceived exertion. Med Sci Sports Exerc 1982; 14: 377-381.

22 Guyatt GH, Berman LB, Townsend M, et al. A measure of quality of life for clinical trials in chronic lung disease. Thorax 1987; 42: 773-778.

23 Zigmond AS, Snaith RP. The hospital anxiety and depression scale. Acta Psychiatr Scand 1983; 67: 361-370.

24 Ellis B, Ries AL. Upper extremity exercise training in pulmonary rehabilitation. J Cardiopulm Rehabil 1991; 11: 227-231.

25 Pepin V, Laviolette L, Brouillade C, et al. Significance of changes in endurance shuttle walking performance. Thorax 2011; 66: 115-120.

26 Vickers AJ, Altman DG. Analysing controlled trials with baseline and follow up measurements. Br Med J 2001; 323: 1123-1124.

27 Singh SJ, Jones PW, Evans R, et al. Minimum clinically important improvement for the incremental shuttle walking test. Thorax 2008; 63: 775-777.

28 Becker BE. Aquatic therapy: scientific foundations and clinical rehabilitation applications. PM R 2009; 1: 859-872.

29 Butcher SJ, Jones RL. The impact of exercise training intensity on change in physiological function in patients with chronic obstructive pulmonary disease. Sports Med 2006; 36: 307-325. 